\title{
Effect of quasibound states on coherent electron transport in twisted nanowires
}

\author{
Giampaolo Cuoghi \\ Centro S3, CNR-Istituto Nanoscienze, Via Campi 213/A, I-41100 Modena, Italy and Dipartimento di Fisica, \\ Università degli Studi di Modena e Reggio Emilia, Modena, Italy
}

\author{
Andrea Bertoni* \\ Centro S3, CNR-Istituto Nanoscienze, Via Campi 213/A, I-41100 Modena, Italy
}

\author{
Andrea Sacchetti \\ Dipartimento di Matematica Pura ed Applicata, Università degli Studi di Modena e Reggio Emilia, Modena, Italy
} (Received 3 November 2010; revised manuscript received 30 March 2011; published 28 June 2011)

\begin{abstract}
Quantum transmission spectra of a twisted electron waveguide expose the coupling between traveling and quasibound states. Through a direct numerical solution of the open-boundary Schrödinger equation, we single out the effects of the twist and show how the presence of a localized state leads to a Breit-Wigner or a Fano resonance in the transmission. We also find that the energy of quasibound states is increased by the twist, despite the constant section area along the waveguide. While the mixing of different transmission channels is expected to reduce the conductance, the shift of localized levels into the traveling-states energy range can reduce their detrimental effects on coherent transport.
\end{abstract}

DOI: 10.1103/PhysRevB.83.245439

PACS number(s): 73.23.Ad, 03.65.-w, 73.63.Nm

\section{INTRODUCTION}

Conductance spectra of quasi-one-dimensional (1D) semiconductor structures display many features that directly expose the quantum nature of carrier transport and are of great interest both for applications and for fundamental understanding. ${ }^{1,2}$ Even in the simplest noninteracting carriers approach, the departure from a constant section of the wire gives rise to complex resonance patterns in the quantum transmission. This originates from the coherent coupling of the energy spectra of different sub-bands and from the interplay of traveling and localized states. ${ }^{3}$ Indeed, the case of a discrete energy spectrum merged with a continuum one, was considered by $\mathrm{Fano}^{4}$ in his seminal work on inelastic-scattering amplitudes of electrons. In that case, the two Hamiltonians with discrete and continuous spectra were that of the electronic degree of freedom of an atom and that of a free electron, respectively. It was shown that the coupling induced by the Coulomb interaction led to a peculiar asymmetric shape of the scattering probability and a discontinuity of the scattering phase. This behavior of the scattering amplitude is now identified in many atomic, ${ }^{5}$ optical, ${ }^{6}$ and transport ${ }^{7}$ experiments (for a review, see Ref. 8).

Here, we analyze the zero-temperature coherent transmission of a quantum waveguide (QW) locally twisted, as depicted in Fig. 1, with the twist inducing a coupling between the sub-bands related to different transverse modes. We adopt a noninteracting carrier model. A local potential, modeling, e.g., the effect of impurities, is also included. For argument's sake, we assume that such a potential is of an attractive kind, thus, generating a discrete set of bound states and Fano resonances in the transmission spectra: The latter will expose the energy of quasibound ${ }^{9}$ states of the twisted QW. For energies close to the above levels, trapping phenomena occur, leading to a reduction of the conductance as an effect of the local trapping potential. In this paper, we will show how this effect is partially negated by the twist. We stress that our results are representative of a more general case, as, for example, a carrier scattered through a quantum dot embedded in a QW or a QW whose Hamiltonian is not separable in the transverse and longitudinal directions, leading to localized states.

The effect of twisting on the conductance is twofold. On one side, it is expected to reduce the conductance. ${ }^{10,11}$ On the other side, this reduction can be compensated by a partial destruction of localization effects (e.g., due to external fields or impurities), induced by the twisting itself. In fact, by means of the complex-scaling method, it has been shown ${ }^{12}$ that stable states associated with a trapping potential may become resonant states when the $\mathrm{QW}$ is twisted. In this paper, we numerically compute the real and imaginary parts of such resonances as a function of the twisting parameter in an explicit model, and we prove that, for such a model, the imaginary part of these resonances actually takes a negative value, this indicating that the corresponding states become unstable. Specifically, our results show that the energy of bound states of the quantum well in the longitudinal direction is increased by the twist and, as they enter the continuous-spectrum range of traveling states, they appear in the transmission characteristic as symmetric (Breit-Wigner ${ }^{13}$ ) or asymmetric $\left(\right.$ Fano $\left.^{14}\right)$ resonant peaks, according to the character of the original bound wave function. The width of the resonances is related to the imaginary component of the eigenvalues of the complex-scaled Hamiltonian, ${ }^{15}$ as we detail in the following, and shows a nonmonotonic behavior. A resonant peak may or may not disappear when its energy reaches the transmission channel with the same transverse energy as the original bound state, according to the corresponding bound state in the straight QW. Indeed, the knowledge of the bound states of the straight QW allows one to predict the position and type of transmission resonances in the twisted system. We also conclude that, when a short-range trapping potential is present, its detrimental effect on the conductance can be partially negated by twisting the QW in the spatial region where the external potential is supported.

Our paper is organized as follows. In Sec. II, we describe the model of the twisted QW and, in the following Sec. III, 


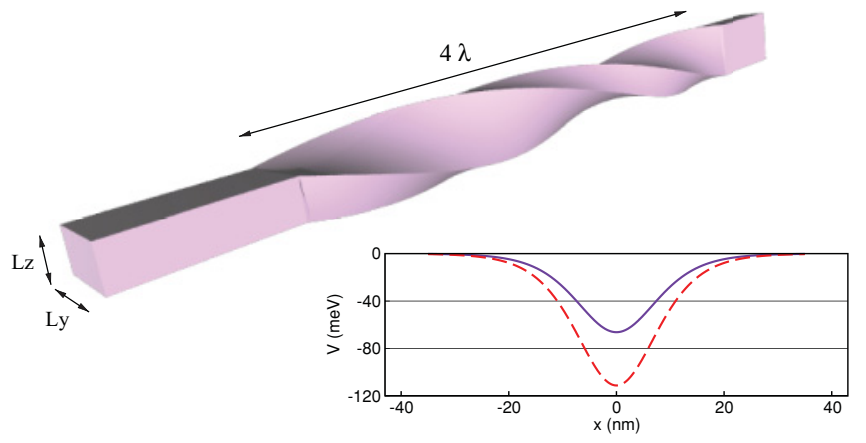

FIG. 1. (Color online) QW with a rectangular cross section $\left(L_{y}=\right.$ $20 \mathrm{~nm}, L_{z}=10 \mathrm{~nm}$ ) twisted for a length of $4 \lambda \simeq 70 \mathrm{~nm}$. Here, the rotation angle is $\Phi=\frac{3}{2} \pi$. (Inset) local confining potential, as given in Eq. (3), with $L_{p}=10 \mathrm{~nm}$ and $v=2.95$ (solid line) or $v=3.95$ (dashed line). The potential well is fully contained in the twisted region.

we outline the real-space numerical approach adopted for the calculation of the scattering states and transmission amplitudes on a non-Cartesian grid. In Sec. IV, the main results of our paper are presented, with particular attention to the evolution of resonant peaks with the QW twist. Finally, in Sec. V, we draw our conclusions. In the final Appendix, analytical details of the complex-scaling approach, mentioned in the main text, are given.

\section{THE PHYSICAL SYSTEM}

We consider a $\mathrm{QW}$ with a rectangular cross section, with a hard-wall confinement. For a straight wire, an elementary solution of the single-band effective-mass Schrödinger equation gives the energy spectrum,

$$
E_{n, k}=E_{n}+\frac{\hbar^{2}}{2 m} k^{2}
$$

with

$$
E_{n}=\frac{\hbar^{2}}{2 m}\left[\left(\frac{n_{y} \pi}{L_{y}}\right)^{2}+\left(\frac{n_{z} \pi}{L_{z}}\right)^{2}\right],
$$

where $m$ is the effective mass of the carriers that we consider isotropic. In fact, here, we neglect atomistic effects and only consider the geometry of the system. Essentially, we are not supposing to take a straight semiconductor wire, fix one end, and rotate the other end by force. Rather, we simply consider a structure with the shape depicted in Fig. 1. $L_{y}$ and $L_{z}$ (with $L_{y} \neq L_{z}$ ) are the thicknesses of the QW in the two directions orthogonal to the current propagation, $k$ is the wave number of the $x$-propagating plane-wave component of the wave function. For the sake of brevity, the sub-band index $n=1,2, \ldots$ (with $E_{n, k} \leqslant E_{(n+1), k}$ ) has been introduced, summarizing the two positive integers $n_{y}$ and $n_{z}$. Since $k$ can be any real number, it is clear that the energy spectrum is a continuum, with $E_{n, k} \in\left[E_{1},+\infty\right)$. Transmission channels, identified by the transverse modes in the two leads, can also be classified according to Eq. (2). As we will show, when the QW is twisted, the above channels are mixed, that is, a scattering state with a single incoming transverse component in the source lead will have many transmitted (reflected) transverse components in the drain (source) lead.

A confining potential well (depicted in the Fig. 1 inset) is introduced along the $x$ direction,

$$
V(x)=\frac{\hbar^{2}}{2 m} \frac{v(v+1)}{L_{p}^{2}}\left[\tanh ^{2}\left(\frac{x}{L_{p}}\right)-1\right],
$$

where the positive parameters $v$ and $L_{p}$ set the depth and the length of the well. Specifically, the minimum of $V$ is $-\hbar^{2} v(v+$ $1) /\left(2 m L_{p}^{2}\right)$, and the region in which $V$ is significantly different from zero is about $6 L_{p}$. We stress that the form given in Eq. (3) has been chosen both to mimic a smooth local confinement and to deal with a potential that has an exact expression for its bound-state eigenvalues: ${ }^{16}$

$$
\mu_{j}=-\frac{\hbar^{2}}{2 m L_{p}^{2}}(\nu+1-j)^{2}, \quad j=1,2, \ldots,\lceil\nu\rceil,
$$

where the ceiling function $\lceil\nu\rceil$ indicates the smallest integer not less than $v$. The above expression is essential to approach the problem analytically through the complex-scaling method that we use to follow the energy vs the twist behavior of the bound states.

The energetic spectrum of the Schrödinger operator for the QW with $V$ consists of a discrete and a continuum part,

$$
\left\{E_{n}+\mu_{j} ; n=1,2, \ldots ; j=1, \ldots,\lceil\nu\rceil\right\} \cup\left[E_{1},+\infty\right) .
$$

For energies above $E_{1}$, the two parts overlap, and eigenvalues of the discrete spectrum are embedded in the continuum spectrum. However, the two subspectra remain well distinct since the system Hamiltonian is separable in a transverse $(y-z$ plane) and a longitudinal ( $x$-direction) component. In fact, if a given energy corresponds to a discrete level and, at the same time, it lies inside the continuum, the corresponding state will be degenerate, with the different eigenfunctions having a different transverse state. In terms of quantum transport along the QW, the above system does not mix different transmission channels or propagating states with bound ones.

Let us now introduce a local twist in the QW. As we will show, this couples different transverse modes, mixing their spectra, and shifts the energy of the discrete states. The deformation adopted, also illustrated in Fig. 1, is a rotation of the rectangular cross section around its center when moving along the $\mathrm{QW}$ axis. A point of coordinates $(x, y, z)$ of the straight QW is transformed according to

$$
\begin{gathered}
x^{\prime}=x, \\
y^{\prime}=y \cos [\varphi(x)]+z \sin [\varphi(x)], \\
z^{\prime}=z \cos [\varphi(x)]-y \sin [\varphi(x)],
\end{gathered}
$$

where

$$
\varphi(x)=\frac{\Phi}{2}\left[\operatorname{erf}\left(\frac{x}{\lambda}\right)+1\right]
$$

is the rotation angle as a function of the longitudinal position $x$. Here, erf is the error function, $\Phi$ is the total rotation angle, and $\lambda$ is a parameter that sets the length of the twisted region. In particular, the QW twist can be considered effective in a length $4 \lambda$ around the origin. Outside the latter region, the QW is essentially straight. 


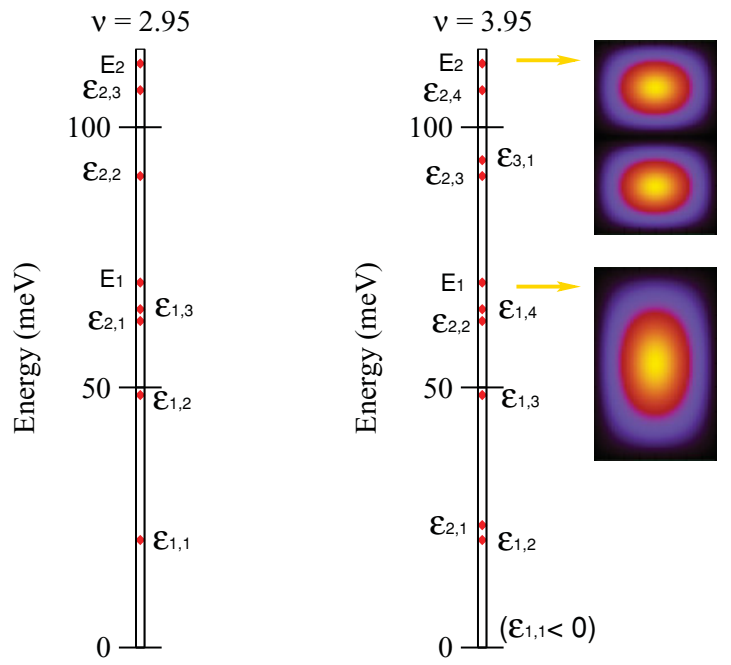

FIG. 2. (Color online) Schematic of the relevant energies of the system without a twist for two different magnitudes $v$ of the quantum well described by Eq. (3). The thresholds of the transport channels, i.e., the transverse modes, are $E_{1}=70.155 \mathrm{meV}, E_{2}=$ $112.248 \mathrm{meV}$, and $E_{3}=182.403 \mathrm{meV}$ (not shown). The energy of a bound state of the straight QW is indicated by $\varepsilon_{n, j}$, with $n$ indicating the transverse mode and $j$ the bound state of $V$ in the longitudinal direction. While the $E_{n}$ 's are fixed, the $\varepsilon_{n, j}$ 's are increased by the twist. Note that, without the twist, all states $\varepsilon_{n, j}$ are bound, while only those that remain under the $E_{1}$ threshold are bound as the twist is applied. The two insets on the right sketch the square modulus of the transverse component of the traveling-wave functions for the first (lower) and second (upper) channels with thresholds $E_{1}$ and $E_{2}$, respectively.

For our simulations, we use GaAs effective electron mass $m=0.067 m_{e}$ and adopt the following set of geometric parameters: $L_{y}=20 \mathrm{~nm}, L_{z}=10 \mathrm{~nm}, \lambda=17.5 \mathrm{~nm}$ (i.e., the twisted region is about $70 \mathrm{~nm})$, and $0 \leqslant \Phi \leqslant 3 \pi$. Two attractive potentials, as given in Eq. (3), have been used, both with $L_{p}=10 \mathrm{~nm}$ (i.e., effective on a length of about $60 \mathrm{~nm}$ around the origin). They differ in their depth: In the first case, $v=2.95$ (corresponding to a minimum of $-66.262 \mathrm{meV}$ ), and in the second case, $v=3.95$ (corresponding to a minimum of $-111.186 \mathrm{meV}$ ). The relative positions of relevant energy levels are reported in Fig. 2 for the two cases. For brevity, the energies of the discrete states are indicated by $\varepsilon_{n, j}=$ $E_{n, 0}+\mu_{j}$ in the following. We stress that the transverse energies $E_{n}$ are fixed, since the cross section is constant, although rotated. On the contrary, the position of $\varepsilon_{n, j}$ depends on the twist, as we will analyze in detail in Sec. IV. In fact, they are the resonant energies that correspond to a local maximum (Breit-Wigner) or a zero (Fano) in the transmission spectra.

\section{NUMERICAL APPROACH}

To obtain the transmission amplitudes of the twisted QW, we solve the three-dimensional (3D) Schrödinger equation with open boundaries through the quantum transmitting boundary method ${ }^{17}$ (QTBM). Electrons are injected from the left lead (see Fig. 1) in a given transverse mode and either can be reflected or can be transmitted to the right lead. With this boundary condition, the differential equation of motion is solved in the internal points of the domain, leading to complex transmission/reflection amplitudes for every channel of the right/left leads. We adopt a curved coordinate system naturally defined by the twist function of Eq. (6), $\mathbf{r}=(x, y, z) \rightarrow \mathbf{r}^{\prime}=$ $\left(x^{\prime}, y^{\prime}, z^{\prime}\right)$. This new coordinate system follows the QW twist and sees a straight QW. However, the equation of motion must also be transformed according to the $\mathbf{r} \rightarrow \mathbf{r}^{\prime}$ relation. In order to do so, we need the metric tensor of the system $\mathbf{G}(\mathbf{r})$ with components $G_{i j}(\mathbf{r})=\left(\partial_{i} \mathbf{r}^{\prime}\right) \cdot\left(\partial_{j} \mathbf{r}^{\prime}\right)$, together with its inverse $\mathbf{G}^{-1}$ with components $G^{i j}$. Here, we used the definitions $\partial_{i}=\partial / \partial x^{i}$ and $(x, y, z)=\left(x^{1}, x^{2}, x^{3}\right)$. In the curved coordinate system, the Hamiltonian reads ${ }^{18,19}$ as follows:

$$
\begin{aligned}
\mathcal{H}(\mathbf{r}) & =-\frac{\hbar^{2}}{2 m} \sum_{i, j=1}^{3} \frac{\partial_{i}}{\sqrt{G}}\left(\sqrt{G} G^{i j} \partial_{j}\right) \\
& =-\frac{\hbar^{2}}{2 m} \sum_{i, j=1}^{3}\left[G^{i j} \partial_{i j}^{2}-\left(\sum_{k, l=1}^{3} G^{k l} \partial_{k l}^{2} \mathbf{r}^{\prime} \cdot G^{i j} \partial_{i} \mathbf{r}^{\prime}\right) \partial_{j}\right]
\end{aligned}
$$

where $G>0$ is the determinant of $\mathbf{G}$ and $\partial_{i j}^{2}=\partial_{i} \partial_{j}$. Note that the coupling of different transmission channels is a direct consequence of the nonzero off-diagonal elements of the metric tensor $G$, used to introduce the twist in the equation of motion. Now, it is easy to define a rectangular mesh following the QW in the new coordinate system and to discretize $\mathcal{H}$ through a finite-difference scheme. Specifically, we adopt a first-order scheme for the partial derivatives and include the leads through an additional self-energy term in the Hamiltonian ${ }^{20}$ whose matrix component coupling the grid points $(x, y, z)$ and $\left(x, y^{\prime}, z^{\prime}\right)$ at the left-lead interface $x$ (fixed) reads

$$
\Sigma_{L}\left(y, z ; y^{\prime}, z^{\prime}\right)=\sum_{n} \chi_{n}(y, z)\left(-i k_{n}\right) \chi_{n}\left(y^{\prime}, z^{\prime}\right),
$$

where $n$ indexes the normal eigenmodes of the rectangularsection leads $\chi_{n}$ and $k_{n}$ is the kinetic wave vector in the $x$ direction. For the right lead, the expression for $\Sigma_{R}$ is similar with the opposite sign. This procedure allows truncating the real-space Hamiltonian matrix and writing the system equation of motion in matrix form as follows:

$$
\left(\begin{array}{ccc}
\Sigma_{L} & t I \ldots 0 & 0 \\
t I & & 0 \\
\vdots & \mathcal{H}-E I & \vdots \\
0 & & t I \\
0 & 0 \ldots t I & \Sigma_{R}
\end{array}\right) \psi=\left(\begin{array}{c}
S \\
0 \\
\vdots \\
0 \\
0
\end{array}\right)
$$

where $t$ represents the coupling strength between an internal point and a point in the leads, $E$ is the total energy of the state $\psi, I$ is an identity matrix, and $S$ is the source term, namely, an array of the same dimension as $\Sigma_{L}$ with elements,

$$
S(y, z)=\sum_{n} A_{n}\left(-i k_{n}\right) \chi_{n}(y, z),
$$

describing the incoming electron wave with amplitude $A_{n}$ in channel $n$. Equation (9) is then solved numerically for a given set of $A_{n}$ and total energy $E$. In fact, the QTBM outlined above takes, as an input, the kinetic energy $E-E_{n}>0$ of the incoming electron and the wave function $\chi_{n}$ of the 
transverse modes $n$ and gives, as an output, the wave function $\psi$ from which the transmission/reflection amplitudes in the different channels ${ }^{21}$ are easily obtained. For this reason, a new calculation must be performed for every $E$ in a chosen set over the range of interest, with $E>E_{n}$.

Actually, in order to find the resonances, we also used a complementary technique: the complex-scaling approach described in the Appendix. ${ }^{12}$ This method leads to a complexeigenvalue problem that allows one to identify, in a straightforward way, the resonances originated by the bound states of $V$. Moreover, it gives the energy levels of bound states below $E_{1}$, not achievable with the QTBM. In fact, the QTBM gives the transmission amplitude of the different transverse modes as a function of the carrier energy, and the position of transport resonances must be detected subsequently, as a relevant peak or a dip in the transmission probability and as a continuum (abrupt) phase shift for a Breit-Wigner (Fano) resonance. However, the complex-scaling method is very demanding from the computational point of view, and we used its results only as a reference for specific cases. We leave the comparison of the two methods for a subsequent paper.

\section{TRANSMISSION SPECTRA}

As anticipated in Sec. II, the transmission spectra of the straight QW can be obtained from a 1D equation of motion with the potential $V$. In order to mix transmission channels, the transverse/longitudinal separability must be lifted. However, a generic deformation of the QW section along the wire not only couples different transverse modes, but also introduces additional resonant energies, as in the case of a closed cavity attached at a side. ${ }^{22,23}$ This can make exposing the sole effect of the coupling between the continuum and the discrete spectra difficult. For this reason, as well as for technological relevance, we choose a kind of deformation that does not alter the shape of the QW cross section, but only its orientation, and does not introduce further resonances. In fact, the QW twist has only two effects: First, it couples the transmission channels so that the transmission probability for a carrier injected in a given transverse mode also has traces of quasibound states of different modes; second, it increases the energies of quasibound states. This can be considered a consequence of the 3D-shape modification of the shallow quantum dot formed by the potential $V$ and the lateral hard-wall confinement. Since the twist is dependent on $x$ and only is applied to the central region, the different wave functions of the quasibound states are affected differently due to their different shapes and peak extensions. The above effects can be seen from the two panels of Fig. 3, where we report the position (top panel) and width (bottom panel) of the resonances in the transmission spectrum of the ground transverse channel as a function of the twist angle. In particular, for each angle $\Phi$, we inject a carrier in the ground transverse mode (with energy $E_{1}$ ) and with several longitudinal kinetic energies from zero to $E_{2}-E_{1}$. From the curves of transmission amplitude vs total energy $E$ (see, e.g., Figs. 4 and 5) we determine the position of the resonances and obtain the imaginary part of the eigenvalue as $-\Gamma / 2$, where $\Gamma$ is the peak or dip width. Note that the above complex eigenvalues correspond to quasibound states with a mean lifetime $\hbar / \Gamma$ on

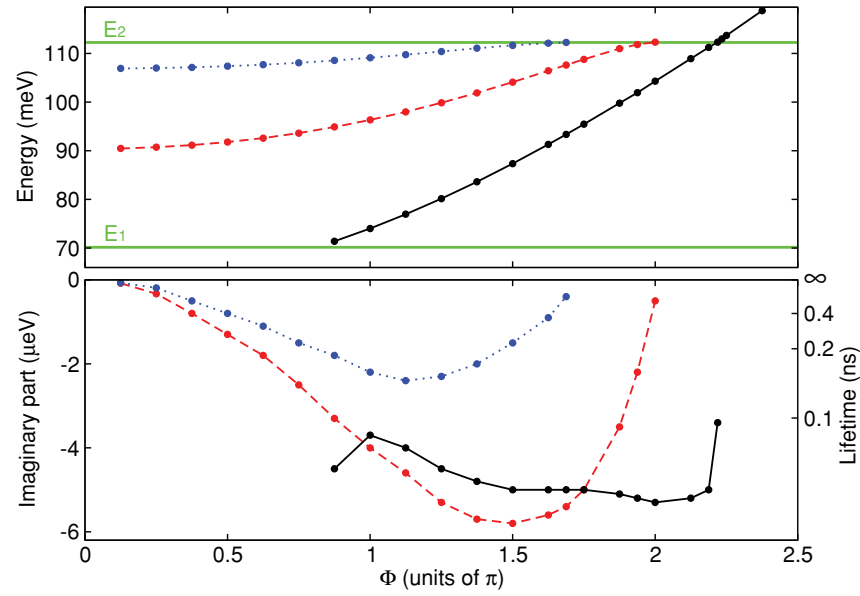

FIG. 3. (Color online) (Top panel) Position of transmission resonances in the ground-mode spectrum as a function of the twist angle $\Phi$, for $v=2.95$. The resonant energies correspond to the real part of the complex-scaled eigenvalues. The threshold energies of the ground and first-excited transverse modes are indicated by the two horizontal lines labeled $E_{1}$ and $E_{2}$, respectively. The three curves correspond to the quasibound states $\epsilon_{2,3}$ (dotted line), $\epsilon_{2,2}$ (dashed line), and $\epsilon_{2,1}$ (solid line). (Bottom panel) Imaginary part of the complex-scaled eigenvalues, corresponding to the half-width of the resonance peaks or dips. While the two Fano resonances $\epsilon_{2,3}$ and $\epsilon_{2,2}$ (see also Fig. 4) disappear as they reach $E_{2}, \epsilon_{2,1}$ is still present in the spectrum at energies exceeding $E_{2}$ in the form of a broad and shallow dip (see also Fig. 5).

the order of a few hundreds of picoseconds, as indicated on the right $y$ axis.

By comparing Fig. 3 with the levels of the straight QW given in Fig. 2, the origin of the two resonances at higher energy (dotted and dashed lines in Fig. 3) is clear. In fact, for $\Phi \sim 0$, only two quasibound levels lie between $E_{1}$ and $E_{2}$, namely, $\epsilon_{2,2}$ and $\epsilon_{2,3}$. They both belong to channel 2 so that, at zero twist, they do not appear in the transmission spectrum,

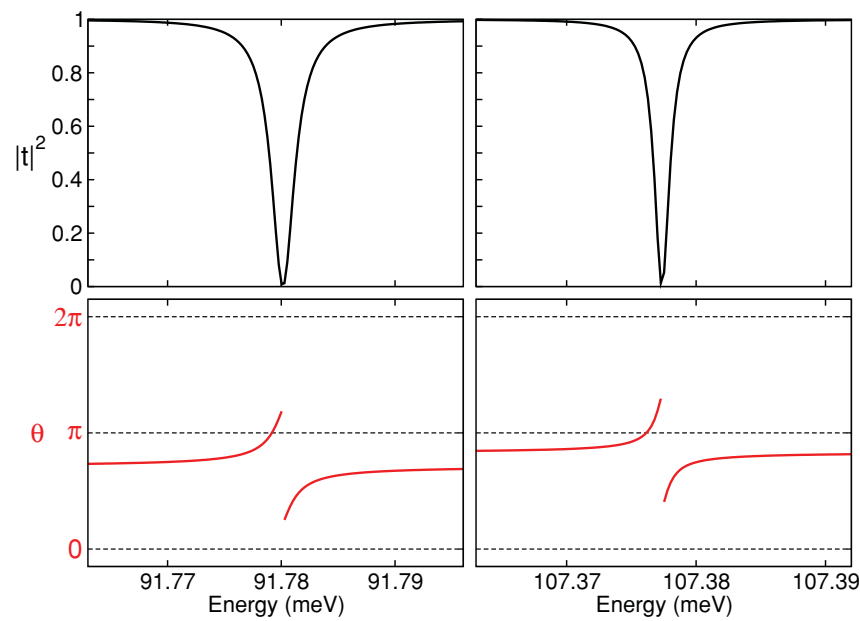

FIG. 4. (Color online) Ground-mode transmission probability (upper panels) and phase (lower panels) around the resonances $\epsilon_{2,2}$ (left panels) and $\epsilon_{2,3}$ (right panels) for a twist angle $\Phi=\pi / 2$. In both cases, phase $\theta$ shows the typical $\pi$ discontinuity of Fano resonances. 


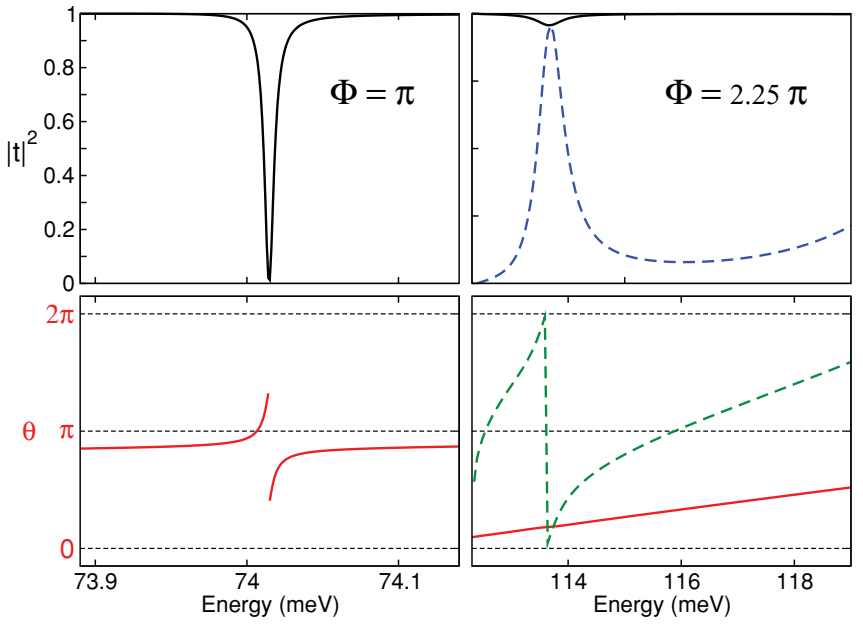

FIG. 5. (Color online) Ground-mode transmission probability (upper panels) and phase (lower panels) around the resonance $\epsilon_{2,1}$ for two different twist angles, namely, $\Phi=\pi$ (left panels) and $\Phi=2.25 \pi$ (right panels, solid line). In the first case, $\epsilon_{2,1}<E_{2}$, and a sharp Fano dip is found. In the second case, $\epsilon_{2,1}>E_{2}$, and its position is only indicated by a very shallow dip. Also, in the latter case, the transmission spectrum of the first-excited mode is reported (dashed line), revealing a pronounced Breit-Wigner resonance.

as can be gathered from the vanishing imaginary part of their eigenvalues in the bottom panel of Fig. 3. As the twist is introduced, the two levels above appear as slightly asymmetric Fano dips in the transmission probability, as reported in the top panels of Fig. 4 for the case of $\Phi=\pi / 2$. However, the Fano character of the resonances is better revealed by the abrupt jump of $\pi$ in the transmission phase $\theta$, as shown in the bottom panels of Fig. 4. The third resonance of Fig. 3 (solid line) appears around $\Phi \simeq 0.85 \pi$ from the low-energy threshold $E_{1}$. Again, it is a Fano resonance, as can be gathered from the left panels of Fig. 5, showing the transmission probability and phase at $\Phi=\pi$. This is confirmed by results of the complexscaling approach, ascribing the resonance to the quasibound level $\epsilon_{2,1}$. In fact, as the twist increases from 0 to $\pi$, both levels $\epsilon_{2,1}$ and $\epsilon_{1,3}$ reach the threshold $E_{1}$. However, while the former shows up as a resonance in the transmission spectrum, the latter disappears as it enters the traveling-states region, with its imaginary part going to zero. Again, this behavior can be traced by the complex-scaling method alone, since the lower energy accessible with the QTBM is $E_{1}$. When $\epsilon_{2,1}$ enters the energy range of the first-mode traveling states, it appears as a Fano resonance since it is a second-mode quasibound state. Moreover, contrary to the other two resonances, it appears with a significant width (bottom panel of Fig. 3 ) from the beginning, since at $\Phi \simeq 0.85 \pi$, the coupling between the modes is already strong.

When the twist increases from $\pi$ to $2 \pi$, the three quasibound levels described above also increase their energy. However, as $\epsilon_{2,2}$ and $\epsilon_{2,3}$ approach the threshold of the second channel, their width decreases, and they finally disappear, with the width going to zero, when their energy reaches $E_{2}$. The behavior of $\epsilon_{2,1}$ is different. In fact, its resonance width is always on the order of $4 \mu \mathrm{eV}$ until the energy reaches $E_{2}$,

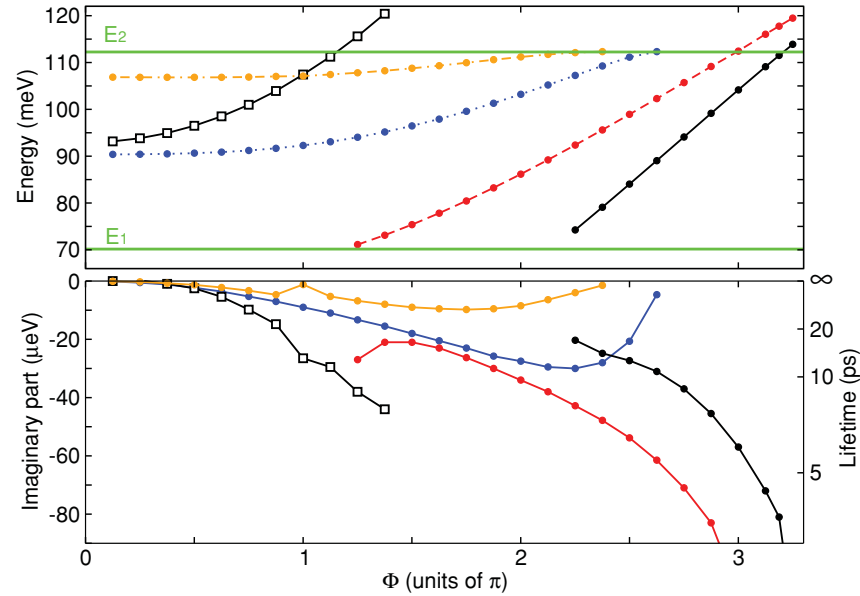

FIG. 6. (Color online) Same as Fig. 3, but for a deeper potential well, with $v=3.95$. Here, four quasibound states with a first-excited transverse mode are present: $\epsilon_{2,1}$ (solid line, with filled circles), $\epsilon_{2,2}$ (dashed line), $\epsilon_{2,3}$ (dotted line), and $\epsilon_{2,4}$ (dot-dashed line), together with a quasibound state with a second-excited transverse mode: $\epsilon_{3,1}$ (solid line with empty squares). The two resonances $\epsilon_{3,1}$ and $\epsilon_{2,4}$, with different transverse modes, cross around $\Phi=\pi$ with a repulsion of their imaginary components. All the resonances in the $\left(E_{1}, E_{2}\right)$ range are of Fano type.

where the width increases by orders of magnitude. This is shown in the right panels of Fig. 5, where the transmission probability (top) and phase (bottom) are shown for a twist $\Phi=$ $2.25 \pi$. Here, $\epsilon_{2,1}>E_{2}$, and the second transmission channel becomes available. The solid line is the first-channel-to-firstchannel transmission and shows a tiny dip at the quasibound state position, reminiscent of the prominent Fano dip of the single-channel case. The dashed line is the second-channel-tosecond-channel transmission, showing a clear Breit-Wigner resonance with the corresponding continuous phase lapse of $\pi .^{24}$ This is not surprising, since in this case, the quasibound state has the same transverse mode of the transmission channel.

The case with $v=3.95$, with a deeper potential well $V$ in the twisted region, presents additional effects. In fact, at zero twist, the energy range between $E_{1}$ and $E_{2}$, where only the ground channel is open, contains bound states of two different transverse modes, namely, the first excited $\left(\epsilon_{2,3}\right.$ and $\left.\epsilon_{2,4}\right)$ and the second excited $\left(\epsilon_{3,1}\right)$, as shown in Fig. 2. As the wire is twisted, the energy of the above three quasibound states increases, as illustrated in the top panel of Fig. 6. However, level $\epsilon_{3,1}$ (solid line with empty squares) increases faster than the other two, it crosses $\epsilon_{2,4}$ around $\Phi=\pi$ and goes beyond $E_{2}$. First of all, we note again that, in the $\left(E_{1}, E_{2}\right)$ range, the transport resonances corresponding to the above quasibound states are Fano resonances, in agreement with the fact that they originate from a transverse mode different from that of the transport channel. This is shown in the left panels of Fig. 7, reporting the transmission probability and phase of the ground channel at $\Phi=\pi$, just after the crossing. At the crossing, we also find a repulsion of the imaginary component of the eigenvalues, visible in the bottom panel of Fig. 6. 


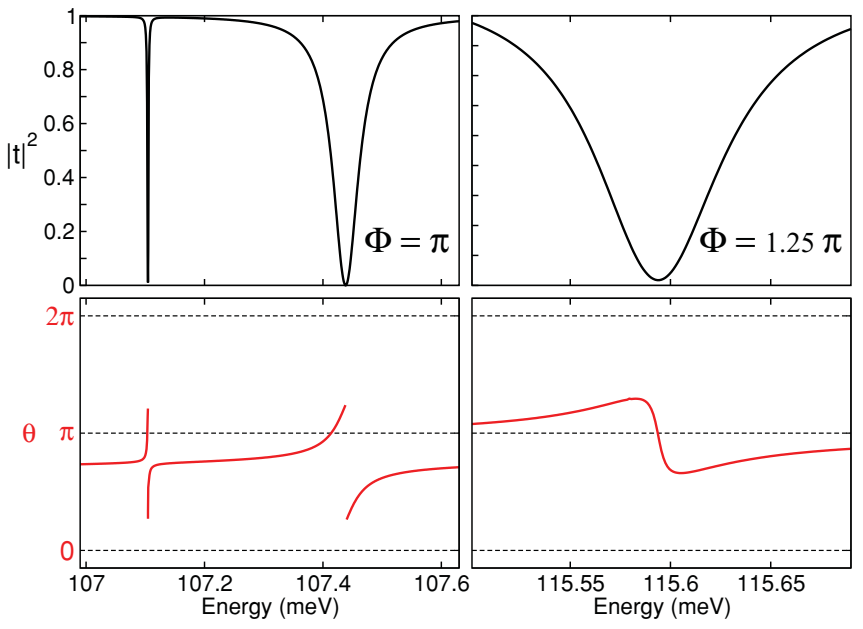

FIG. 7. (Color online) Ground-mode transmission probability (upper panels) and phase (lower panels) showing the resonance $\epsilon_{3,1}$ just after the crossing with $\epsilon_{2,4}$ (at $\Phi=\pi$, left panels) and above the second-mode threshold $E_{2}$ (at $\Phi=1.25 \pi$, right panels). In the first case, the $\pi$ discontinuities of the transmission phase evidence the Fano character of the resonances. In the second case, a third channel is available, and the resonance does not present either Fano or Breit-Wigner character.

When $\epsilon_{3,1}>E_{2}$, i.e., it enters the energy region with two transport channels, it does not disappear, as $\epsilon_{2,3}$ and $\epsilon_{2,4}$ do at a larger twist, but simply changes its characters. Now, the minimum of the dip does not reach zero, and the phase does not present the $\pi$ discontinuity (Fig. 7 , right panels). Here, in fact, two continuum energy spectra and a quasibound level overlap, this lifting the rigid zero-transmission properties of the previous case where a quasibound state is embedded in a single continuum.

As already mentioned, the two resonances $\epsilon_{2,3}$ and $\epsilon_{2,4}$, present in the spectrum since small twist angles, disappear as they reach $E_{2}$, with their width going to zero. Two additional resonances enter the ground-mode region at larger twists: $\epsilon_{2,2}$ and $\epsilon_{2,1}$, represented in Fig. 6 by a dashed line and a solid line, respectively. They are also Fano resonances, but after reaching $E_{2}$, they do not vanish. In fact, their width increases, and their minimum does not reach zero, as shown in Fig. 8 for $\epsilon_{2,2}$. Obviously, in this region, they are also present in the transmission spectrum of the first-excited channel as Breit-Wigner resonances (dashed line in Fig. 8), since their transverse mode is the first-excited one as well. Correspondingly, their transmission phase presents a smooth evolution of $\pi$.

In Fig. 9, we also report the ballistic conductance ${ }^{20}$ vs $E_{F}$ at zero temperature in the range $E_{1}<E_{F}<E_{2}$ (i.e., where only the first channel is active), where $E_{F}+e \frac{\Delta V}{2}$ and $E_{F}-e \frac{\Delta V}{2}$ are the Fermi energy of the left and right leads, respectively. We take two different applied biases, namely, $\Delta V=100 \mu \mathrm{V}$ (solid lines) and $\Delta V=1 \mathrm{mV}$ (dashed lines), for the same two twist angles of Fig. 8. Figure 9 shows that the dips in the conductance should be detectable with a realistic value of source-drain biases and that the twist is able to clear the resonance dips of a portion of the transmission spectrum. Specifically, the range of the figure covers the first transmission

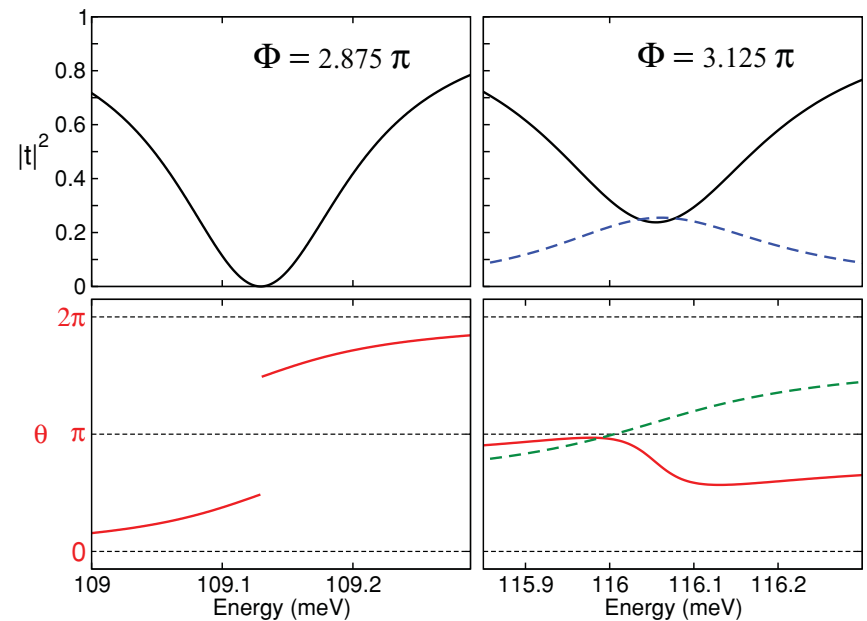

FIG. 8. (Color online) Transmission probability (upper panels) and phase (lower panels) around the resonance $\epsilon_{2,2}$ for the system with $v=3.95$. Two different twist angles are considered, with the resonance close to the second-channel threshold: $\Phi=2.875 \pi$ (left panels), where $\epsilon_{2,2}<E_{2}$ and $\Phi=3.125 \pi$ (right panels), where $\epsilon_{2,2}>E_{2}$. In the second case, both the ground mode (solid line) and the first-excited mode (dashed line) transmission probabilities are reported. As for the case of Fig. 7, after the crossing of $E_{2}$, the $\pi$ discontinuity of the phase is lost due to the availability of a third transmission channel. The broad peak in the first-excited channel is a Breit-Wigner resonance.

channel with two resonance dips in the case with the lower twist (top panel). In the large-twist case (bottom panel), the two dips coming from the quasibound states $\varepsilon_{2,1}$ and $\varepsilon_{2,2}$ have been pushed to the right, with the second one outside the range considered. Furthermore, it is clear from the figure that the twist does not introduce substantial modifications to the conductance, as it keeps its maximum value $\frac{2 e}{h}$ in the range between the first-channel threshold $E_{1}$ and the first dip $\varepsilon_{2,1}$, regardless of the twist.

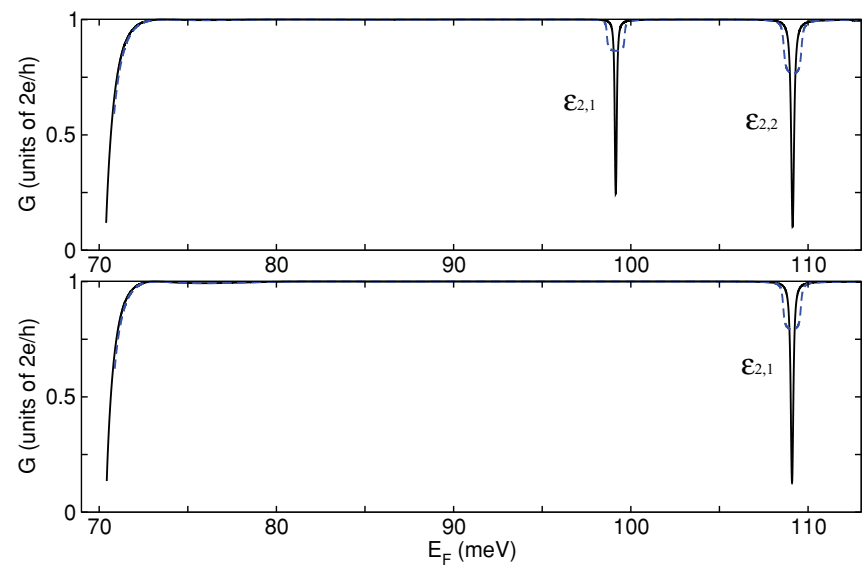

FIG. 9. (Color online) Ballistic conductance at zero temperature vs mean-Fermi energy of source and drain leads for two different applied biases: $100 \mu \mathrm{V}$ (solid lines) and $1 \mathrm{mV}$ (dashed lines). Here, $v=3.95$. Two different twist angles $\Phi=2.875 \pi$ (upper panel) and $\Phi=3.125 \pi$ (lower panels) are considered. 


\section{CONCLUSIONS}

By solving the open-boundary Schrödinger equation through the QTBM, we obtained the transmission spectra of the twisted QW. The effect of the twist can be summarized in the following points. First, the twist is able to mix different transmission channels despite the fact that the transverse QW section is not altered (but only rotated). If no external potential $V$ is present, no evident resonance peak is found in the transmission spectra. In particular, no Fano resonance can exist, since no bound state is present that can become embedded in the continuum portion of the energy spectrum. ${ }^{25}$ If the attractive potential $V$ is considered, its bound states are coupled to traveling states and appear as resonance peaks/dips in the transmission spectra. However, no additional resonances are introduced with respect to the straight case. Second, the character of the resonance depends on the transverse mode of the original bound state. In fact, when the latter is equal to the transverse mode of the transmission channel, we find a Breit-Wigner resonance, otherwise, we find a Fano resonance. In case more than two channels are available, we do not find the $\pi$ discontinuity of the transmission phase typical of Fano resonances. From a different perspective, the twist allows controlling whether, at a given injection energy, a quasibound state affects the conductance through a Breit-Wigner resonance peak or a Fano resonance dip. Third, the twist increases the energy of quasibound levels. The higher the transverse mode of the quasibound state, the faster its energy increases. However, the change of the resonances width is nonmonotonic with the twist. In general, it increases from zero when the energy of the bound state is already in the transport region in the straight QW and decreases as the above energy reaches the threshold of the transmission channel with the same transverse mode as the quasibound state. Fourth, resonances that are present from the beginning in the first-channel region disappear as they reach $E_{2}$, while resonances that enter the $E_{1}-E_{2}$ region at a finite twist, persist in the multichannel region. The strict behavior described above could help in anticipating the characters of transmission spectra of QW locally twisted once the spectra of the straight wire are known. Furthermore, it supports the idea that the twist can reduce the effects of localized states on quantum conductance, since it shifts their levels toward higher energies, possibly beyond Fermi level of the quasi-1D nanostructure.

As a last comment, we note that, in a realistic experimental setup, operating in a multielectron regime, electron-electron interaction would likely have a large impact on the transmission spectra up to concealing the noninteracting single-particle features reported above. In fact, our paper focuses on the role of the QW twist on the interplay between discrete and continuum spectra of the system Schrödinger equation and on its effect on the transmission probability of the twisted-wire potential layout. A sensible comparison between our transmission spectra and the quantum conductance of a real device would require a strict single-electron occupancy of the device with densities on the order of $10^{15}$ particles $/ \mathrm{cm}^{3}$ or less and an accurate tailoring of the energy of the injected electrons, e.g., by means of a resonant tunnel diode structure. In the few-electron limit (i.e., with an average number of electrons in the device between 2 and about 12) correlation effects can be important, ${ }^{26}$ while in the many-electron regime (with a high electron density on the order of $10^{18}$ particles $/ \mathrm{cm}^{3}$ or higher), intercarrier Coulomb interaction can be included in the model in a Hartree approximation through a self-consistent Schrödinger-Poisson procedure. The self-consistent potential will eventually cause a realignment of the band profile in order to follow the source and drain Fermi levels. Moreover, for low values of the twist, the bound states of the potential well $V(x)$ that lie below the lower threshold $E_{1}$ would be occupied. On one hand, since these states are not embedded in the continuum spectrum, they do not take part in the formation of the transmission resonances. On the other hand, the Coulomb repulsion between the bound electrons and the traveling one would substantially modify the profile of the QW conduction band.

\section{ACKNOWLEDGMENT}

We thank P. Bordone, G. Ferrari, and H. Kovařík for most helpful discussions.

\section{APPENDIX: COMPLEX-SCALING METHOD}

So far, we have identified resonant energies with singular points of the reflection/transmission coefficient. In the framework of the complex-scaling method, introduced in the 1970s by Aguilar and Combes ${ }^{27}$ and Baslev and Combes ${ }^{28}$ (for a review, see also Ref. 29), resonances were identified with the complex eigenvalues of a nonsymmetric linear operator obtained from the original one by analytic complex deformation. The real part of such complex-valued eigenvalues coincides with the usual resonance energy level, while the imaginary part is associated with the resonant state lifetime. The complex-scaling method has been employed in Ref. 12 to twisted QW in order to prove the existence of resonances, and here, we briefly resume it.

As already mentioned in the main text, we use the complex-scaling method in order to support our findings by means of an approach complementary to the QTBM. In fact, the application of complex-scaling to nanowire transport is very powerful since it allows accessing energy regimes below the lower traveling state. These regimes are precluded to standard methods for open systems, such as the QTBM.

Let $\omega=\left(-\frac{1}{2} L_{y},+\frac{1}{2} L_{y}\right) \times\left(-\frac{1}{2} L_{z},+\frac{1}{2} L_{z}\right) \subset R^{2}$ be the rectangular cross section of our QW. For a given $x \in R$ and $(y, z) \in \omega$, we define a generalized mapping of the kind given in Eq. (6), where $\varphi(x)=\Phi \alpha(x)$ and where $\alpha: R \rightarrow R$ is a differentiable function that represents the twisting and $\Phi \geqslant 0$ is a real-valued parameter that represents the strength of the twist. In order to ease a direct comparison, in this Appendix, we use the symbols already defined in the main text, even if the formalism used here is of more general validity. Let $\Omega_{\Phi}$ be the twisted QW, and let

$$
H_{\Phi}=-\frac{\hbar^{2}}{2 m} \Delta+V(x)
$$

be the time-independent Schrödinger operator on $\Omega_{\Phi}$, that is, the wave function $\psi$ belongs to $L^{2}\left(\Omega_{\Phi}\right)$ with Dirichlet boundary conditions at $\partial \Omega_{\Phi} . V$ represents the external 
potential Eq. (3), depending only on the longitudinal variable $x$. In the following, for the sake of definiteness, let us assume the unit choice such that $\frac{\hbar^{2}}{2 m}=1$.

In order to analyze the operator $H_{\Phi}$, we go back to the untwisted tube $\Omega$. The operator $H_{\Phi}$ then takes the form $K_{\Phi}$,

$K_{\Phi}=-\partial_{y y}^{2}-\partial_{z z}^{2}-\left[\partial_{x}+\Phi \alpha^{\prime}(x) \partial_{\tau}\right]^{2}+V(x)=K_{0}+U_{\Phi}$,

where

$$
\partial_{\tau}=y \partial_{z}-z \partial_{y}
$$

and

$$
K_{0}=-\partial_{x x}^{2}-\partial_{y y}^{2}-\partial_{z z}^{2}+V(x)
$$

and

$$
\begin{aligned}
U_{\Phi} & =-\left[\partial_{x}+\Phi \alpha^{\prime}(x) \partial_{\tau}\right]^{2}+\partial_{x x}^{2} \\
& =-2 \Phi \alpha^{\prime}(x) \partial_{x \tau}^{2}-\Phi \alpha^{\prime \prime}(x) \partial_{\tau}-\Phi^{2}\left[\alpha^{\prime}(x)\right]^{2} \partial_{\tau \tau}^{2} .
\end{aligned}
$$

The operator $K_{\Phi}$ is a symmetric operator on $L^{2}(\Omega)$ with Dirichlet boundary conditions at $\partial \Omega$. The spectrum of $K_{0}$ is given by Eq. (5), that is, the spectrum of $K_{0}$ admits embedded eigenvalues in the continuous spectrum. In Ref. 12, it has been proved that such embedded eigenvalues become resonances when we add the perturbation $U_{\Phi}$ to $K_{0}$. Resonances are defined by employing the method of exterior complex scaling to the operator $K_{\Phi}$, provided that the potential $V$ is a bounded potential that extends to an analytic function with respect to $x$ in some sector and the twisting function $\alpha(x)$ extends to an analytic function with respect to $x$ in a suitable complex set. The exterior complex-scaling method consists of introducing the mapping $S_{\theta}$, which acts as a complex dilation in the longitudinal variable $x$,

$$
\left(S_{\theta} \psi\right)(x, y, z)=e^{\theta / 2} \psi\left(e^{\theta} x, y, z\right), \quad \theta \in C, \quad \operatorname{Im} \theta>0 .
$$

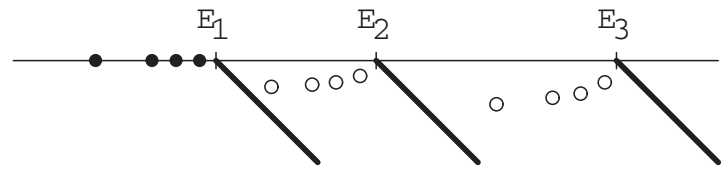

FIG. 10. The essential spectrum of $K_{\Phi}(\theta)$ is given by the half-lines $E_{n}+e^{-2 i \operatorname{Im} \theta} R^{+}$(full lines). The eigenvalues of $K_{\Phi}(\theta)$ (denoted by open circles) with a strictly negative imaginary part are the resonances of $H_{\Phi}$; for energies below the threshold $E_{1}$, the eigenvalues of $K_{\Phi}(\theta)$ (denoted by full circles) are purely real valued, and they are eigenvalues of $H_{\Phi}$.

The transformed operator is not a symmetric operator, and it takes the form

$$
K_{\Phi}(\theta)=S_{\theta} K_{\Phi} S_{\theta}^{-1}=K_{0}(\theta)+U_{\Phi}(\theta),
$$

where

$$
K_{0}(\theta)=S_{\theta} K_{0} S_{\theta}^{-1}=-e^{-2 \theta} \partial_{x x}^{2}-\partial_{y y}^{2}-\partial_{z z}^{2}+V\left(e^{\theta} x\right),
$$

and

$$
\begin{aligned}
U_{\Phi}(\theta)= & S_{\theta} U_{\Phi} S_{\theta}^{-1} \\
= & -2 \Phi e^{-\theta} \alpha^{\prime}\left(e^{\theta} x\right) \partial_{x \tau}^{2}-\Phi \alpha^{\prime \prime}\left(e^{\theta} x\right) \partial_{\tau} \\
& -\Phi^{2}\left[\alpha^{\prime}\left(e^{\theta} x\right)\right]^{2} \partial_{\tau \tau}^{2} .
\end{aligned}
$$

Then, the essential spectrum of $K_{\Phi}(\theta)$ consists of the sequence of the half-lines (Fig. 10) $E_{n}+e^{-2 i \operatorname{Im} \theta} R^{+}, n=$ $1,2, \ldots$, and, by a standard argument, it turns out that the eigenvalues of $K_{\Phi}(\theta)$ are analytic functions of $\theta$, they are, in fact, independent of $\theta$. These nonreal eigenvalues of $K_{\Phi}(\theta)$, for $\theta$ such that $\operatorname{Im} \theta>0$, are identified with the resonances of $K_{\Phi}$ (and, hence, with the resonances of $\left.H_{\Phi}\right){ }^{29}$ *andrea.bertoni@unimore.it

${ }^{1}$ T. Ihn, Electronic Quantum Transport in Mesoscopic Semiconductor Structures (Springer, New York, 2004).

${ }^{2}$ R. Rurali, Rev. Mod. Phys. 82, 427 (2010).

${ }^{3}$ J. U. Nöckel and A. D. Stone, Phys. Rev. B 50, 17415 (1994).

${ }^{4}$ U. Fano, Phys. Rev. 124, 1866 (1961).

${ }^{5}$ J. Fransson and A. V. Balatsky, Phys. Rev. B 75, 153309 (2007).

${ }^{6}$ S. Rotter, F. Libisch, J. Burgdörfer, U. Kuhl, and H.-J. Stöckmann, Phys. Rev. E 69, 046208 (2004).

${ }^{7}$ J. Göres, D. Goldhaber-Gordon, S. Heemeyer, M. A. Kastner, H. Shtrikman, D. Mahalu, and U. Meirav, Phys. Rev. B 62, 2188 (2000).

${ }^{8}$ A. E. Miroshnichenko, S. Flach, and Y. S. Kivshar, Rev. Mod. Phys. 82, 2257 (2010).

${ }^{9}$ In the present paper, we use the term bound state for an eigenstate of the system Schrödinger equation belonging to $L^{2}$ space, while quasibound states are resonant states, associated with complex poles of the scattering matrix whose imaginary part is strictly negative (and related to the state lifetime). A traveling state is a bounded non-normalizable solution (i.e., $\notin L^{2}$ ) of the system Schrödinger equation.
${ }^{10}$ H. Suzuura, Physica E (Amsterdam) 34, 674 (2006).

${ }^{11}$ S.-H. Tseng, N.-H. Tai, M.-T. Chang, and L.-J. Chou, Carbon 47, 3472 (2009).

${ }^{12}$ H. Kovařík and A. Sacchetti, J. Phys. A: Math. Theor. 40, 8371 (2007).

${ }^{13}$ G. Breit and E. Wigner, Phys. Rev. 49, 519 (1936).

${ }^{14}$ U. Fano, Nuovo Cimento 12, 154 (1935).

${ }^{15}$ W. P. Reinhardt, Annu. Rev. Phys. Chem. 33, 223 (1982).

${ }^{16} \mathrm{P}$. M. Morse and H. Feshbach, Methods of Theoretical Physics (McGraw-Hill, New York, 1953), Vol. II.

${ }^{17}$ C. S. Lent and D. J. Kirkner, J. Appl. Phys. 67, 6353 (1990).

${ }^{18}$ R. C. T. da Costa, Phys. Rev. A 23, 1982 (1981).

${ }^{19}$ G. Ferrari and G. Cuoghi, Phys. Rev. Lett. 100, 230403 (2008).

${ }^{20}$ S. Datta, Electronic Transport in Mesoscopic Systems (Cambridge University Press, Cambridge, UK, 1995).

${ }^{21}$ G. Cuoghi, G. Ferrari, and A. Bertoni, Phys. Rev. B 79, 073410 (2009).

${ }^{22}$ P. Price, IEEE Trans. Electron Devices 39, 520 (1992).

${ }^{23}$ Z. S. W. Porod and C. Lent, Appl. Phys. Lett. 61, 1350 (1992).

${ }^{24}$ Note that, in Fig. 5, the abscissa of the right panels has a scale much larger than in the left panels or in Fig. 4. As a consequence, the 
peaks/dip reported here have widths much larger than the previous case, as described in the text. Also note that the transmission phase $\theta$ has been reduced to the $[0,2 \pi]$ region so that a value of $2 \pi+a$ is rescaled and is reported as $a$. The vertical dashed line is not a phase jump: On the contrary, it has been introduced to indicate a continuity of the curve, as opposed to the Fano case of the left panel.

${ }^{25}$ The lack of resonance peaks does not mean that no channel mixing is present. Indeed, the twist considered in this paper depends on the transport direction $x$, and the system lacks translational symmetry: Input/output transport channels are mixed, also without $V$.

${ }^{26}$ A. Bertoni and G. Goldoni, Phys. Rev. B 75, 235318 (2007).

${ }^{27}$ J. Aguilar and J. M. Combes, Commun. Math. Phys. 22, 269 (1971).

${ }^{28}$ E. Balslev and J. M. Combes, Commun. Math. Phys. 22, 280 (1971).

${ }^{29}$ H. Cycon, R. G. Froese, W. Kirsch, and B. Simon, Schrödinger Operators: With Application to Quantum Mechanics and Global Geometry (Springer-Verlag, Berlin, 1987). 